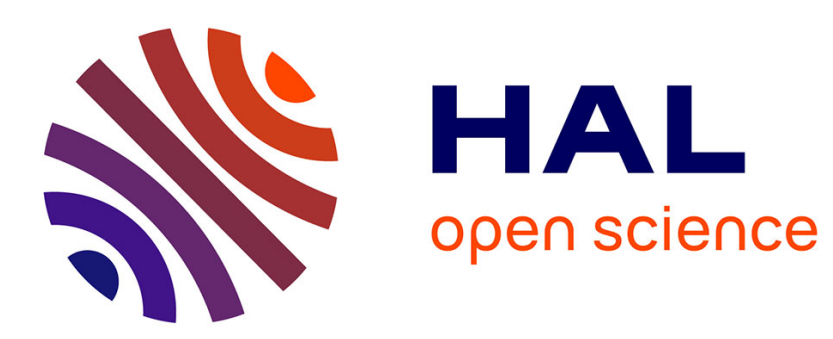

\title{
Chaotic characteristic of a linear oscillator coupled with vibro-impact nonlinear energy sink
}

\author{
Tao Li, Claude-Henri Lamarque, Sébastien Seguy, Alain Berlioz
}

\section{To cite this version:}

Tao Li, Claude-Henri Lamarque, Sébastien Seguy, Alain Berlioz. Chaotic characteristic of a linear oscillator coupled with vibro-impact nonlinear energy sink. Nonlinear Dynamics, 2018, 91 (4), pp.23192330. 10.1007/s11071-017-4015-y . hal-01750700

\section{HAL Id: hal-01750700 \\ https://hal.science/hal-01750700}

Submitted on 3 Dec 2018

HAL is a multi-disciplinary open access archive for the deposit and dissemination of scientific research documents, whether they are published or not. The documents may come from teaching and research institutions in France or abroad, or from public or private research centers.
L'archive ouverte pluridisciplinaire HAL, est destinée au dépôt et à la diffusion de documents scientifiques de niveau recherche, publiés ou non, émanant des établissements d'enseignement et de recherche français ou étrangers, des laboratoires publics ou privés. 


\title{
Chaotic characteristic of a linear oscillator coupled with vibro-impact nonlinear energy sink
}

\author{
Tao Li · Claude-Henri Lamarque · Sébastien Seguy · Alain Berlioz
}

Received: date / Accepted: date

\begin{abstract}
The chaotic characteristic of a system with vibroimpact nonlinear energy sink is studied here. An analytical method is developed to calculate Lyapunov exponent. The mechanism by which impact results in chaos is further clarified rather only the calculation of Lyapunov exponent. In addition, an approach to identify Lyapunov exponents from experimental data is proposed, and the estimated results are consistent with numerical results.
\end{abstract}

Keywords Chaos - Lyapunov exponent - Vibro impact · Nonlinear energy sink · Targeted energy transfer · Impact damper

\section{Introduction}

Nonlinear systems with impact have been extensively studied for decades [1]. Recently, vibro-impact phenomenon has been re-examined from the viewpoint of targeted energy transfer [2]. As a result, its dynamics is further investigated with the application of new analytical methods [3-6] and even experimental observations [7-12]. Impact is proved to be efficient to control vibration and its corresponding device

Tao Li

Institut Clément Ader (ICA), CNRS-INSA-ISAE-Mines Albi-UPS, Université de Toulouse, 3 rue Caroline Aigle, 31400, Toulouse, France

E-mail: tli@insa-toulouse.fr or li_tao_lumiere@126.com

Sébastien Seguy

E-mail: sebastien.seguy@insa-toulouse.fr

Alain Berlioz

E-mail: alain.berlioz@univ-tlse3.fr

Claude-Henri Lamarque

Université de Lyon, École Nationale des Travaux Publics de l'État, LTDS UMR CNRS 5513, 3 rue Maurice Audin, 69518 Vaulx-en-Velin

Cedex, France

E-mail: claude.lamarque@entpe.fr is termed as vibro-impact (VI) nonlinear energy sink (NES), meanwhile the existence of impact can result in complicated dynamics. For example, the strongly modulated characteristic is experimentally observed from the response of acceleration in [9] and also observed from the response of displacement in [10]. The intermittent feature is well demonstrated by the experimentally observed impact time difference [9]. Although many aspects of dynamics are further studied, whether the above mentioned characteristic is related to chaos is not verified.

In $[5,6]$, the strongly modulated response (SMR) of a linear system coupled with VI NES is proved to be chaotic. Actually, the characteristics of modulation and chaos are two different aspects of a single response. The mentioned study is an attempt to combine these two aspects. From the viewpoint of comparing different characteristics, this attempt is pioneering, but this study is not complete for two reasons. Firstly, only the results of Lyapunov exponents are showed and the method to calculate them is not displayed. Secondly, Lyapunov exponent is only an average measure of the divergent or convergent characteristic of two initial close orbits. The relation between impact and chaos should be further explained with more details. Since it is the addition of impact that makes such features possible, the common role of impact that results in different features should be found.

Impact phenomenon is a typical member of nonlinear phenomenon with discontinuity. In the aspect of Lyapunov exponent, a general method to calculate it for dynamic systems with discontinuities is developed by Muller [13] and the transfer matrix at the instants of impact is corrected. With a similar idea as Muller, the chaos of a forced pendulum with friction is studied in [14]. Around chaos resulting from impact, there are already many studies [15-18]. Their common objective is to calculate Lyapunov exponent in order to judge a response is chaos or not, as has been done for other nonlinear systems, though their methods are different. 


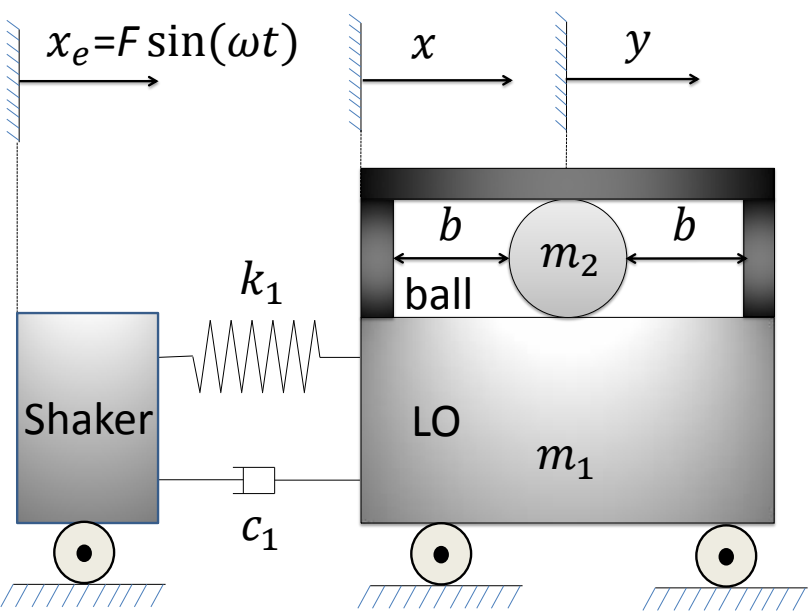

Fig. 1 Schema of a LO coupled with a VI NES under periodic excitation

However, the objective of this paper is not only to calculate Lyapunov exponent, but also to find the factor that contributes to positive Lyapunov exponent. Evidently, it comes from impact, but the specific mechanism is not evident and should be further explored. More specifically, it is to find a more detailed measure of chaos rather than only such a common measure as Lyapunov exponent. That is to say, the role of impact should be directly observed, if chaos is caused by impact. Similarly, the role of friction should be observed if friction is the cause of chaos. In addition, the possibility of identification of Lyapunov exponents from experimental data will be explored here.

The paper is organized as follows. In Section 2, the model of a vibro-impact system is presented. In Section 3, the analytical derivation to calculate Lyapunov exponents is treated. In Section 4, some numerical results are presented. In Section 5, a method is proposed to identify Lyapunov exponents from experimental data. Finally, conclusion of this paper summarizes the main results.

\section{Modeling the vibro-impact system}

The considered vibro-impact system is displayed in Fig. 1 $[7,9,10]$. A linear oscillator $(\mathrm{LO})$ is periodically driven by a shaker. A VI NES is coupled with LO only through impact, and the friction between them is neglected.

During periods without impacts, the system is governed by the following equation:

$$
\begin{aligned}
& \ddot{x}+\varepsilon \lambda \dot{x}+x=\varepsilon G \sin \Omega \tau+\varepsilon^{2} \lambda G \Omega \cos \Omega \tau \\
& \varepsilon \ddot{y}=0 \\
& \forall|x-y|<b
\end{aligned}
$$

and corresponding parameters are as follows:

$$
\begin{aligned}
& \varepsilon=\frac{m_{2}}{m_{1}}, \quad \omega_{0}^{2}=\frac{k_{1}}{m_{1}}, \quad f_{0}=\frac{\omega_{0}}{2 \pi}, \quad \tau=\omega_{0} t, \\
& \lambda=\frac{c_{1}}{m_{2} \omega_{0}}, \quad \Omega=\frac{\omega}{\omega_{0}}, \quad G=\frac{F}{\varepsilon}
\end{aligned}
$$

where $x, m_{1}, c_{1}$ and $k_{1}$ are the displacement, mass, damping and stiffness of the LO, respectively. $y$ and $m_{2}$ are displacement and mass of VI NES, respectively. The dots denote differentiation with respect to dimensionless time $\tau$. $b$ represents the clearance. $x_{e}$ is the displacement imposed on the base by shaker. $\varepsilon G \sin \Omega \tau$ and $\varepsilon^{2} \lambda_{1} G \Omega \cos \Omega \tau$ represent the contribution of force by displacement and velocity of shaker, respectively.

Eq. (1) can also be written as:

$$
\begin{aligned}
& \ddot{x}+\varepsilon \lambda \dot{x}+x=\varepsilon A \sin (\Omega \tau+\phi) \\
& \varepsilon \ddot{y}=0 \\
& \forall|x-y|<b
\end{aligned}
$$

where

$$
\begin{aligned}
A & =\sqrt{G^{2}+\lambda^{2} \varepsilon^{2} G^{2} \Omega^{2}} \\
\phi & =\arctan (\lambda \varepsilon \Omega)
\end{aligned}
$$

When $|x-y|=b$, impacts occur. The relation between after and before impact is obtained under the hypothesis of the simplified shock theory and the condition of total momentum conservation:

$$
\begin{aligned}
& x^{+}=x^{-}, \quad y^{+}=y^{-} \\
& \dot{x}^{+}+\varepsilon \dot{y}^{+}=\dot{x}^{-}+\varepsilon \dot{y}^{-}, \quad \dot{x}^{+}-\dot{y}^{+}=-R\left(\dot{x}^{-}-\dot{y}^{-}\right), \\
& \text {for }|x-y|=b
\end{aligned}
$$

where $R$ is the restitution coefficient, and the superscripts + and - indicate time immediately after and before impact.

\section{Analytical treatment to calculate Lyapunov exponent}

The objective here is to derive formula to calculate the Lyapunov exponents. Correspondingly, it is to calculate the transfer matrix between impacts and the transfer matrix at the moments of impacts.

The state variables of flow $\Phi$ can be expressed as:

$\Phi\left(\tau_{0}, x_{0}, \tau\right)=\left[\begin{array}{l}x_{1}(\tau) \\ x_{2}(\tau) \\ x_{3}(\tau) \\ x_{4}(\tau)\end{array}\right]=\left[\begin{array}{c}\dot{x} \\ x \\ \dot{y} \\ y\end{array}\right]$

then, 


$$
\begin{aligned}
& \dot{\Phi}=f(\Phi) \\
& =\left[\begin{array}{cccc}
-\varepsilon \lambda & -1 & 0 & 0 \\
1 & 0 & 0 & 0 \\
0 & 0 & 0 & 0 \\
0 & 0 & 1 & 0
\end{array}\right] \Phi+\left[\begin{array}{c}
\varepsilon A \sin (\Omega \tau+\phi) \\
0 \\
0 \\
0
\end{array}\right]
\end{aligned}
$$

If initial conditions are given:

$$
\Phi\left(\tau_{0}, x_{0}, \tau=0\right)=\left[\begin{array}{l}
x_{1}(0) \\
x_{2}(0) \\
x_{3}(0) \\
x_{4}(0)
\end{array}\right]=\left[\begin{array}{l}
x 10 \\
x 20 \\
x 30 \\
x 40
\end{array}\right]
$$

The equation of motion between impacts can be obtained and expressed as follows:

$$
\begin{aligned}
& x_{1}(\tau)=\dot{x_{2}}(\tau) \\
& x_{2}(\tau)= \\
& \mathrm{e}^{-\varepsilon \lambda\left(\tau-\tau_{0}\right)}\left(p 1 \sin \left(\omega_{d}\left(\tau-\tau_{0}\right)\right)+q 1 \cos \left(\omega_{d}\left(\tau-\tau_{0}\right)\right)\right) \\
& +f 1 \sin \left(\Omega\left(\tau-\tau_{0}\right)\right)+f 2 \cos \left(\Omega\left(\tau-\tau_{0}\right)\right) \\
& x_{3}(\tau)=\dot{x_{4}}(\tau) \\
& x_{4}(\tau)=x 30\left(\tau-\tau_{0}\right)+x 40
\end{aligned}
$$

where $\tau_{0}$ is the initial time and

$$
\begin{aligned}
& f 1=-\frac{\varepsilon A\left[\left(\Omega^{2}-1\right) \cos (\phi)-2 \lambda \varepsilon \Omega \sin (\phi)\right]}{\Omega^{4}+\left(4 \varepsilon^{2} \lambda^{2}-2\right) \Omega^{2}+1} \\
& f 2=-\frac{\varepsilon A\left[\left(\Omega^{2}-1\right) \sin (\phi)+2 \lambda \varepsilon \cos (\phi) \Omega\right]}{\Omega^{4}+\left(4 \varepsilon^{2} \lambda^{2}-2\right) \Omega^{2}+1}
\end{aligned}
$$

$$
\text { and }
$$

$$
\begin{aligned}
& q 1=x 20-f 2 \\
& p 1=-\frac{-x 10-\varepsilon \lambda x 20+\varepsilon \lambda f 2+f 1 \Omega}{\omega_{d}}
\end{aligned}
$$

and

$\omega_{d}=\sqrt{1-\varepsilon^{2} \lambda^{2}}$

Now, the $i^{t h}$ impact is studied, $i=1,2,3 \cdots$. The corresponding time is $\tau_{i}$ with $\tau_{i}^{-}$and $\tau_{i}^{+}$denoting the moment before and after this impact.

$$
\left(\tau_{i}\right) \leq \tau<\tau_{i+1} \quad \dot{\Phi}=f(\Phi), \quad \Phi\left(\tau_{i}\right)=\Phi_{i}
$$

To calculate Lyapunov exponents, an initial difference of states is needed:

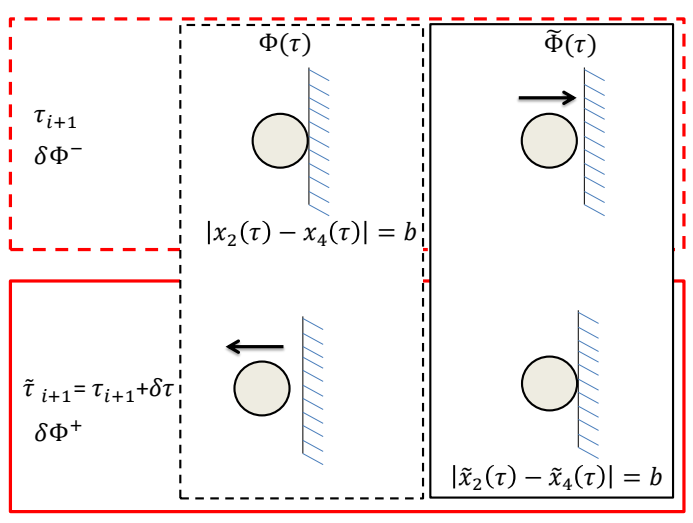

Fig. 2 Trajectory difference before impacts $\left(\delta \Phi^{-}\right)$and after impacts $\left(\delta \Phi^{+}\right)$: original impact in black rectangle (thin broken line) at time $\tau_{i+1}$ in red rectangle (thick broken line), perturbed impact in black rectangle (thin line) at time $\widetilde{\tau}_{i+1}$ in red rectangle (thick broken line)

$\delta \Phi(\tau)=\widetilde{\Phi}(\tau)-\Phi(\tau)=\left[\begin{array}{l}\delta x_{1}(\tau) \\ \delta x_{2}(\tau) \\ \delta x_{3}(\tau) \\ \delta x_{4}(\tau)\end{array}\right]$

where

$\widetilde{\Phi}(\tau)=\left[\begin{array}{c}\widetilde{x_{1}}(\tau) \\ \widetilde{x_{2}}(\tau) \\ \widetilde{x_{3}}(\tau) \\ \widetilde{x_{4}}(\tau)\end{array}\right], \quad \Phi(\tau)=\left[\begin{array}{l}x_{1}(\tau) \\ x_{2}(\tau) \\ x_{3}(\tau) \\ x_{4}(\tau)\end{array}\right]$

are state variables of perturbed trajectory and original trajectory, respectively. For the impact of the original motion $\left(\tau_{i+1}\right)$ and that of perturbed motion $\left(\widetilde{\tau}_{i+1}\right)$, the whole process is demonstrated in Fig. 2. The objective here is to calculate the transfer matrix between the trajectory difference at the moment just before this first impact $\left(\tau_{i+1}\right)$ and that just after the moment of the second impact $\left(\widetilde{\tau}_{i+1}\right)$.

$\delta \Phi\left(\tau_{i}^{+}\right)$after the $i^{t h}$ impact is:

$\delta \Phi\left(\tau_{i}^{+}\right)=\left[\begin{array}{l}\delta x_{1}\left(\tau_{i}^{+}\right) \\ \delta x_{2}\left(\tau_{i}^{+}\right) \\ \delta x_{3}\left(\tau_{i}^{+}\right) \\ \delta x_{4}\left(\tau_{i}^{+}\right)\end{array}\right]$

For the period between the $i^{\text {th }}$ and $(i+1)^{t h}$ impact, the evolution of differences is governed and can be obtained by the following equation:

$\left(\tau_{i}\right) \leq \tau<\tau_{i+1}: \quad \delta \dot{\Phi}=F(\tau) \delta \Phi$

where the starting time is $\tau_{i}^{+}$. With Eq. (6), 
$F(\tau)=\left.\frac{\partial f}{\partial \Phi^{T}}\right|_{\Phi=\Phi(\tau)}=\left[\begin{array}{cccc}-\varepsilon \lambda & -1 & 0 & 0 \\ 1 & 0 & 0 & 0 \\ 0 & 0 & 0 & 0 \\ 0 & 0 & 1 & 0\end{array}\right]$

With Eqs. (16) and (17),

$$
\delta \Phi(\tau)=J 1_{i}(\tau) \delta \Phi\left(\tau_{i}^{+}\right)
$$

where

$J 1_{i}(\tau)=\left[\begin{array}{cccc}a_{11} & a_{12} & 0 & 0 \\ a_{21} & a_{22} & 0 & 0 \\ 0 & 0 & 1 & 0 \\ 0 & 0 & \tau-\tau_{i} & 1\end{array}\right]$

and

$$
\begin{aligned}
& a_{11}=\mathrm{e}^{-\varepsilon \lambda\left(\tau-\tau_{i}\right)}\left[\cos \left(\omega_{d}\left(\tau-\tau_{i}\right)\right)-\frac{\varepsilon \lambda}{\omega_{d}} \sin \left(\omega_{d}\left(\tau-\tau_{i}\right)\right)\right] \\
& a_{12}=-\frac{\mathrm{e}^{-\varepsilon \lambda\left(\tau-\tau_{i}\right)}\left(\varepsilon^{2} \lambda^{2}+\omega_{d}^{2}\right)}{\omega_{d}} \sin \left(\omega_{d}\left(\tau-\tau_{i}\right)\right) \\
& \left.a_{21}=\frac{\mathrm{e}^{-\varepsilon \lambda\left(\tau-\tau_{i}\right)} \sin \left(\omega_{d}\left(\tau-\tau_{i}\right)\right)}{\omega_{d}} \sin \left(\omega_{d}\left(\tau-\tau_{i}\right)\right)+\cos \left(\omega_{d}\left(\tau-\tau_{i}\right)\right)\right] \\
& a_{22}=\mathrm{e}^{-\varepsilon \lambda\left(\tau-\tau_{i}\right)}\left[\frac{\varepsilon \lambda}{\omega_{d}} \sin \right)
\end{aligned}
$$

The above four coefficients of matrix are not influenced by excitation.

Therefore, the transfer matrix from the moment $\left(\tau_{i}^{+}\right)$to the moment $\left(\tau_{i+1}^{-}\right)$can be obtained:

$J_{i}=J 1_{i}\left(\tau_{i+1}^{-}\right)$

Then the transfer matrix at the moment of impact as showed in Fig. 2 is considered. The impact condition is the same for both original and perturbed motions, and can be expressed as follows:

$g(\tau)=\left|x_{2}(\tau)-x_{4}(\tau)\right|-b$

Then to continue the calculation, suppose $x_{2}(\tau)>x_{4}(\tau)$, it just specifies one approaching direction of impact,

$G(\Phi)=\left.\frac{\partial g(\Phi)}{\partial \Phi^{T}}\right|_{\Phi=\Phi(\tau)}=\left[\begin{array}{llll}0 & 1 & 0 & -1\end{array}\right]$

At the moment of impact $\tau_{i+1}$, the state variables between $\tau_{i+1}^{+}$and $\tau_{i+1}^{-}$meet: $\left[\begin{array}{l}x_{1}\left(\tau_{i+1}^{+}\right) \\ x_{2}\left(\tau_{i+1}^{+}\right) \\ x_{3}\left(\tau_{i+1}^{+}\right) \\ x_{4}\left(\tau_{i+1}^{+}\right)\end{array}\right]=S\left[\begin{array}{l}x_{1}\left(\tau_{i+1}^{-}\right) \\ x_{2}\left(\tau_{i+1}^{-}\right) \\ x_{3}\left(\tau_{i+1}^{-}\right) \\ x_{4}\left(\tau_{i+1}^{-}\right)\end{array}\right]$

where

$S=\left[\begin{array}{cccc}\frac{1-R \varepsilon}{\varepsilon+1} & 0 & \frac{\varepsilon+R \varepsilon}{\varepsilon+1} & 0 \\ 0 & 1 & 0 & 0 \\ \frac{1+R}{\varepsilon+1} & 0 & \frac{-R+\varepsilon}{\varepsilon+1} & 0 \\ 0 & 0 & 0 & 1\end{array}\right]$

$S$ is supposed to be time invariant and is the same at both sides of impacts. According to the work of Muller [13], the following equation can be obtained:

$\delta \Phi\left(\tau_{i+1}^{+}\right)=M_{i} \times \delta \Phi\left(\tau_{i+1}^{-}\right)$

where

$M_{i}=S-\frac{\left[S \dot{\Phi}\left(\tau_{i+1}^{-}\right)-\dot{\Phi}\left(\tau_{i+1}^{+}\right)\right] G\left(\tau_{i+1}^{-}\right)}{G\left(\tau_{i+1}^{-}\right) \dot{\Phi}\left(\tau_{i+1}^{-}\right)}$

where

$G\left(\tau_{i+1}^{-}\right) \dot{\Phi}\left(\tau_{i+1}^{-}\right)=x 1\left(\tau_{i+1}^{-}\right)-x 3\left(\tau_{i+1}^{-}\right)$

If $x_{2}(\tau) \leq x_{4}(\tau)$, Eq. (27) still holds. Let

$\delta \Phi\left(\tau_{i+1}^{+}\right)=T_{i} \times \delta \Phi\left(\tau_{i}^{+}\right)$

where

$T_{i}=M_{i} \times J_{i}$

when $i=1, \delta \Phi\left(\tau_{i}^{+}\right)=\delta \Phi(0)$. Here the starting time of calculation is fixed to the first impact moment and it will not influence the ultimate goal of the calculation of Lyapunov exponents.

For a period of time $T e$, we suppose there are $\mathrm{n}$ impacts. At the end of $n^{\text {th }}$ impact:

$\delta \Phi\left(\tau_{n}^{+}\right)=M_{n} \times J_{n} \cdots M_{i} \times J_{i} \cdots M_{1} \times J_{1} \times \delta \Phi(0)$

Let $M\left(\tau_{i}\right)$ be the transfer matrix of the former $i$ impact:

$M\left(\tau_{i}\right)=M_{i} \times J_{i} \cdots M_{1} \times J_{1}$

Let $u_{i}(i=1 . .4)$ be the eigenvalue of transfer matrix $M\left(\tau_{i}\right)$ and $\lambda_{k}(k=1 . .4)$ be the Lyapunov exponent.

$\lambda_{k}=\frac{1}{\tau_{i}-\tau_{0}} \ln \left(\left|u_{i}\right|\right)$

where $\tau_{0}$ is the starting time. Through the calculation of $\lambda_{k}$, chaotic performance can be judged from an average viewpoint. 
Table 1 Simulation parameters

\begin{tabular}{|c|c|c|c|}
\hline \multicolumn{4}{|c|}{ Physical Parameters } \\
\hline $\begin{array}{c}m_{1} \\
k_{1} \\
R \\
\end{array}$ & $\begin{array}{c}3.807 \mathrm{~kg} \\
11.68 * 10^{3} \mathrm{~N} / \mathrm{m} \\
0.6\end{array}$ & $\begin{array}{c}c_{1} \\
m_{2}\end{array}$ & $\begin{array}{c}2.53 \mathrm{Ns} / \mathrm{m} \\
32 \mathrm{~g}\end{array}$ \\
\hline \multicolumn{4}{|c|}{ Reduced Parameters } \\
\hline$\varepsilon$ & $0.84 \%$ & $\lambda$ & 1.43 \\
\hline \multicolumn{4}{|c|}{ Excitation Parameters } \\
\hline$G$ & 0.02 & $\Omega$ & 1 \\
\hline \multicolumn{4}{|c|}{ Initial conditions } \\
\hline$x(0)$ & 0 & $\dot{x}(0)$ & 0 \\
\hline$y(0)$ & $95 \% b$ & $\dot{y}(0)$ & 0 \\
\hline
\end{tabular}

\section{Numerical results}

Here, numerical results related to the calculation of transient Lyapunov exponent are demonstrated.

According to former studies [9], response regimes can be classified according to the average number of impacts per cycle, and it is noted as $z$. Here, six categories are showed and described as follows and almost all of them have been experimentally observed.

Type I represents sparse impact without periods of two impacts per cycle and the distribution of impacts is irregular, namely there is only one impact during many cycles. Type II represents SMR with intermittent periods of two impacts per cycle. There are desynchronized parts and synchronized parts. When $z$ is closer to two, the duration of synchronized parts becomes longer. Type III represents regimes with two symmetrical impacts per cycle. Type IV represents regimes with two asymmetrical impacts per cycle. Type $\mathrm{V}$ represents other periodic regimes with integer times of impacts per cycle (e.g., three impacts per cycle), symmetrical or asymmetrical. Type VI represents other response regimes with irregular distribution of impacts. Among them, chaotic behavior have been observed for Types I, II and VI.

To start our calculation, the parameters in Table 1 are used and only $b$ is varied to obtain different response regimes.

\subsection{Type II: chaotic SMR}

Firstly, the results of a SMR with $b=0.022 \mathrm{~m}$ is showed. The characteristic of a response can be reflected from different viewpoints. All results here are selected only around the calculation of Lyapunov Exponent, and more information can be found in $[9,10]$.

In Fig. 3, the strongly modulated characteristic is well demonstrated by the envelop of displacement. For every peak of $x$, LO and VI NES move in desynchronization in the increasing part and in synchronization in the decreasing part, i.e., two impacts per cycle. The impact number per cycle

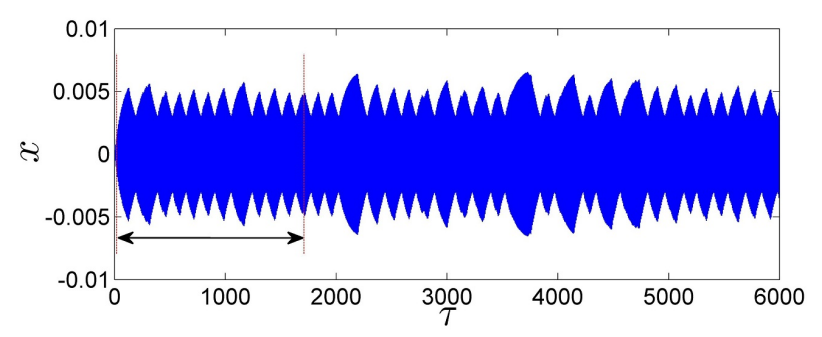

Fig. 3 Type II and SMR with $b=0.022 \mathrm{~m}$ : displacement $x$ of LO

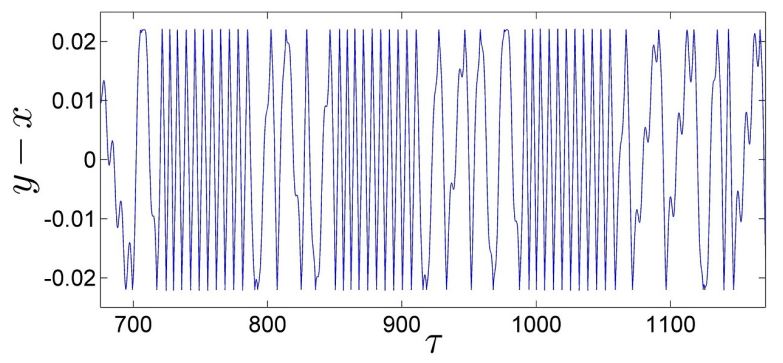

Fig. 4 Type II and SMR with $b=0.022 \mathrm{~m}$ : enlarged view of relative displacement $y-x$

Table 2 Transient Lyapunov exponents at different time periods

\begin{tabular}{ccc}
\hline$\left[\tau_{s}, \tau_{e}\right]$ & $\lambda_{1}$ & $\lambda_{3}$ \\
\hline$[18.13001708 .9]$ & 0.1093 & 0.08427 \\
{$[1708.93652 .7]$} & 0.1143 & 0.09242 \\
{$[3652.75521 .3]$} & 0.1197 & 0.1015 \\
\hline
\end{tabular}

is better displayed by relative displacement in Fig. 4 and is equal to the number of extreme values per cycle. In the sparse parts, the amplitude of $x$ increases with $z<2$. In the dense parts, the amplitude of $x$ decreases with $z=2$.

To calculate Lyapunov exponents, we just calculate one period of limited time as showed by the two broken red line in Fig. 3, considering that too large value of transfer matrix $M$ in Eq. (32) will exceed the capability of computer. In this period, the transient response has disappeared and it should be enough for the calculation of Lyapunov exponents. The evolution of four Lyapunov exponents is showed in Fig. 5. These four transient Lyapunov exponents are almost constant and are positive, which denotes the chaotic characteristic.

However, the evolution of Lyapunov exponents is not smooth, and sometimes there are transient spikes. They result from the calculation of $u_{i}$ in (33). At these non-smooth points, $u_{i}$ is equal or close to zero. Relevant impact points are probably related to the grazing bifurcation but cannot be justified with evidence here. As a result, the calculation error is greatly increased. Nevertheless, the general trend is right and can still predict the evolution of Lyapunov exponents.

To test the influence of the calculation time, different starting and ending times are chosen as showed in Table 2. 


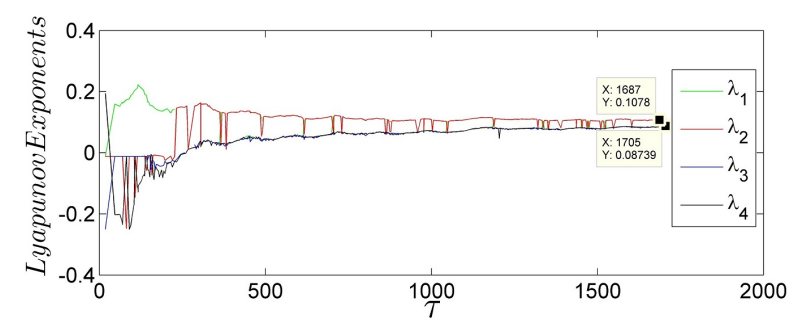

Fig. 5 Type II and SMR with $b=0.022 \mathrm{~m}$ : evolution of four Lyapunov exponents (a)

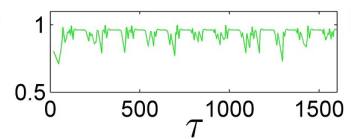

(c)

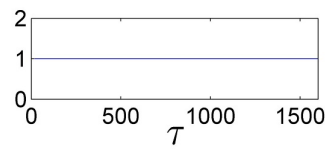

(b)

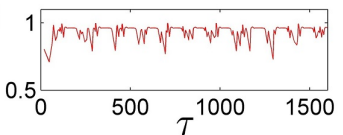

(d)

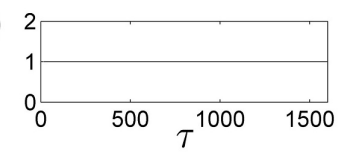

Fig. 6 Type II and SMR with $b=0.022 \mathrm{~m}$ : absolute value of four eigenvalues of matrix $J_{i}$
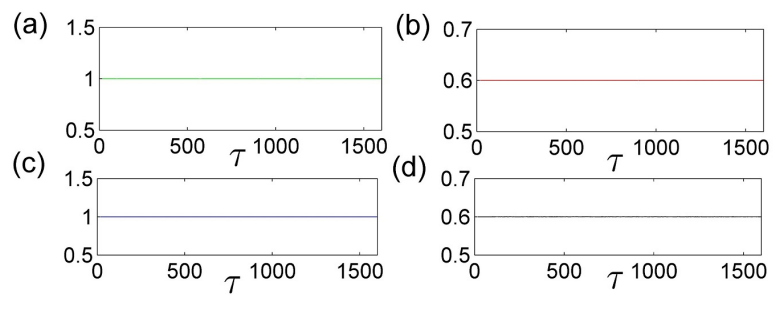

Fig. 7 Type II and SMR with $b=0.022 \mathrm{~m}$ : absolute value of four eigenvalues of matrix $M_{i}$

Although $\lambda_{1}$ and $\lambda_{3}$ vary a little, their difference are small and their sign are always positive. The small difference seems reasonable since every selected time history of displacement is different.

Then, the eigenvalues of matrix $J_{i}$ in Eq. (21) without influence of impacts is calculated, and their absolute values are obtained and showed in Fig. 6. The initial difference of LO will decrease with two values less than one as showed in Fig. 6(a-b). This is quite natural since LO is a dissipative system governed by Eq. (6) during these periods without impacts. And it is also natural to observe two unit values for VI NES in Fig. 6(c-d) since it is a conservative system itself.

Then, the eigenvalues of matrix $M_{i}$ in Eq. (27) at the impact moments are calculated. From the results showed in Fig. 7, the initial difference of displacement will not be changed during the impact process by themselves. On the contrary, the initial difference of velocity will be decreased by impact with a reduction coefficient $R$ by themselves. Since only the values related to eigenvalues are showed here, the interaction between these four states once impacts are not clear. The latter probably could result in any possible chaos. (a)

(c)
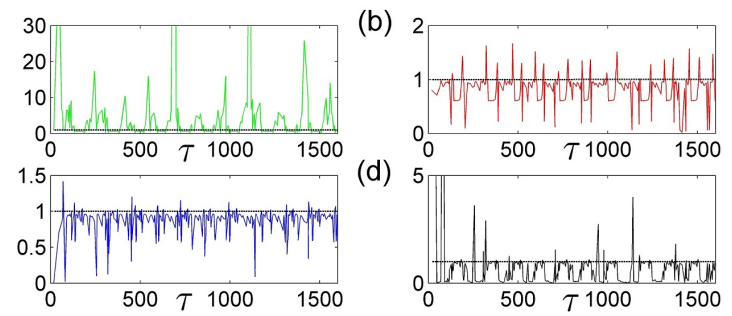

Fig. 8 Type II and SMR with $b=0.022 \mathrm{~m}$ : absolute value of four eigenvalues of matrix $T_{i}$

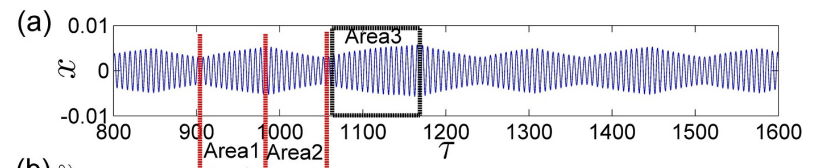

(b)

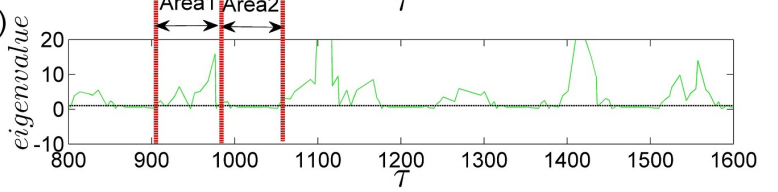

Fig. 9 Type II and SMR with $b=0.022 \mathrm{~m}$ : comparison of the time history of strongly modulated displacement and the evolution of eigenvalues of matrix $T_{i}$. (a) Displacement $x$; (b) one of the eigenvalues of matrix $T_{i}$

To further observe the influence of impact, the eigenvalues of matrix $T_{i}$ in Eq. (30) is calculated. In this way, the interaction of these four states can be observed. As showed in Fig. 8, these two absolute values of eigenvalues of matrix sometimes become very large and greater than one.

Then we compare these eigenvalues with corresponding time history of displacement as showed in Fig. 9, and only the first eigenvalue is demonstrated in Fig. 9(b). It is seen that the eigenvalue is greater than one in areal and less than one in area2. Here, area1 and area2 are two typical examples of desynchronization and synchronization. For the synchronization parts, the eigenvalue has almost the same value, but this value changes a lot in the desynchronization parts. In area3, the largest value is observed and it has a close relation to the maximal amplitude of modulated displacement.

Evidently, the chaotic characteristic results from impact, but only the existence of impact will not definitively result in chaos. From the above results, chaos results from irregular impacts, namely during desynchronization between LO and VI NES. Since a Lyapunov exponent reflects an average trend, the results here demonstrate more details and reveal the essence of chaos. About this point, it will be further verified from the following results.

\subsection{Other types}

Type I: the evolution of Lyapunov exponents with $b=0.04$ $\mathrm{m}$ is showed in Fig. 10, four positive values are obtained. 
Therefore, the irregular and occasional impacts can result in chaos.

(a)

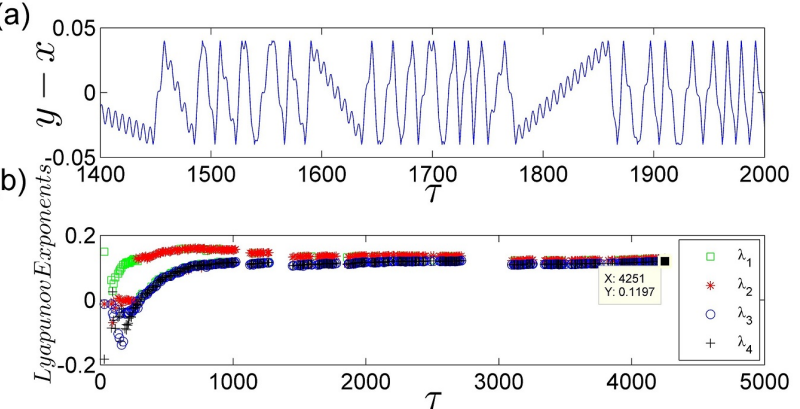

Fig. 10 Type I with $b=0.04 \mathrm{~m}$ : evolution of Lyapunov exponents. (a) Relative displacement $y-x$; (b) transient Lyapunov exponents

Type III and IV: the results for $b=0.015 \mathrm{~m}$ and $b=$ $0.008 \mathrm{~m}$ are showed in Fig. 11 and Fig. 12, respectively. The former is with two symmetrical impacts and the latter asymmetrical. For these two, four negative values (two of them almost zero) are obtained. Therefore, they are not chaos and the regular impact does not generate chaotic behavior.

(a)

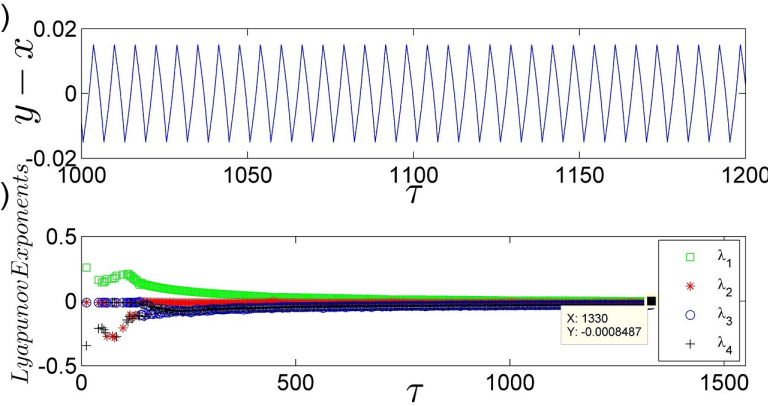

Fig. 11 Type III with $b=0.015 \mathrm{~m}$ : evolution of Lyapunov exponents. (a) Relative displacement $y-x$; (b) transient Lyapunov exponents

(a)

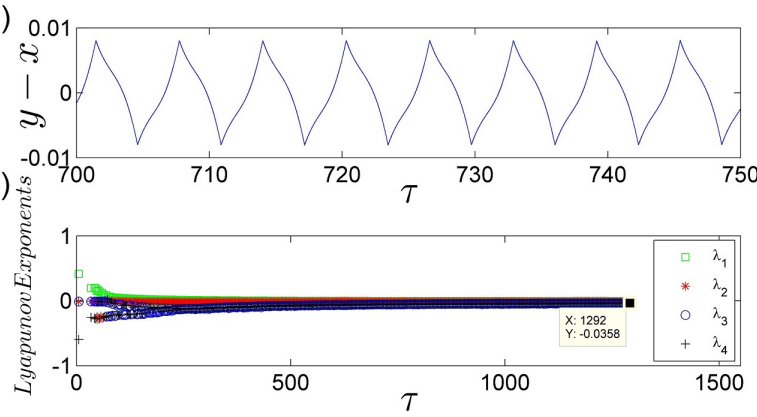

Fig. 12 Type IV with $b=0.008 \mathrm{~m}$ : evolution of Lyapunov exponents. (a) Relative displacement $y-x$; (b) transient Lyapunov exponents
Table 3 Transient Lyapunov exponents of different regimes

\begin{tabular}{ccc}
\hline$b$ & $\lambda_{1}$ & $\lambda_{3}$ \\
\hline \multicolumn{3}{c}{ Type I } \\
\hline 0.04 & 0.1321 & 0.1245 \\
0.034 & 0.1372 & 0.127 \\
\hline \multicolumn{3}{c}{ Type II: SMR } \\
\hline 0.031 & 0.1528 & 0.1391 \\
0.028 & 0.1452 & 0.1309 \\
0.025 & 0.1138 & 0.09727 \\
0.022 & 0.1093 & 0.08427 \\
0.020 & 0.1014 & 0.0783 \\
0.018 & 0.06368 & 0.03913 \\
0.017 & 0.04703 & 0.02043 \\
0.0165 & 0.03686 & 0.01084 \\
\hline
\end{tabular}

Then $b$ is decreased to $0.004 \mathrm{~m}$, three impacts per cycle is obtained, namely Type $\mathrm{V}$. The evolution of eigenvalues is showed in Fig. 13 and four negative values are also obtained.

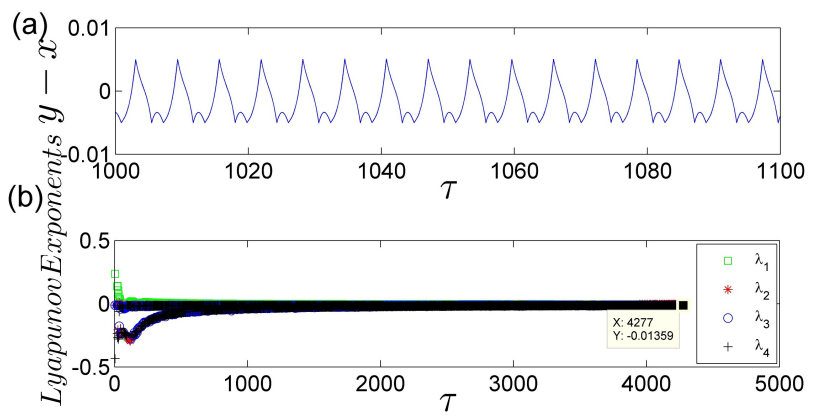

Fig. 13 Type $\mathrm{V}$ with $b=0.004 \mathrm{~m}$ : evolution of Lyapunov exponents. (a) Relative displacement $y-x$; (b) transient Lyapunov exponents

When $b$ is further increased, the impact number is between 3 and 4, as showed in Fig. 14(a). For this irregular response, the final Lyapunov exponents are positive.

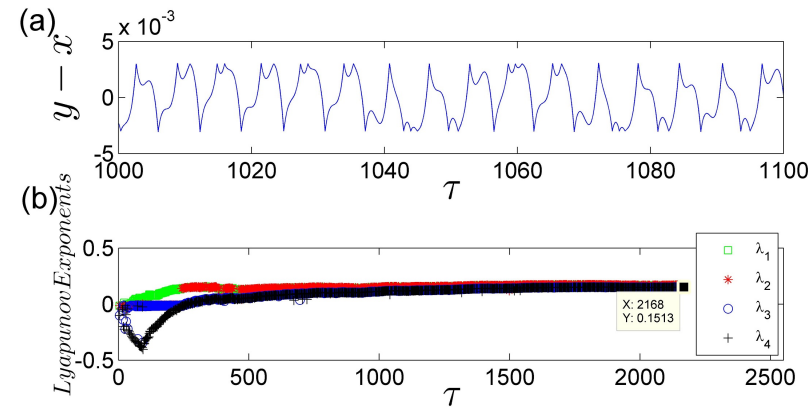

Fig. 14 Type VI with $b=0.003 \mathrm{~m}$ : evolution of Lyapunov exponents. (a) Relative displacement $y-x$; (b) transient Lyapunov exponents

If the value of positive Lyapunov exponents is related to the irregular impact, different SMRs with different duration 
Table 4 Experimental Parameters [9]

\begin{tabular}{cccc}
\hline \multicolumn{4}{c}{ Physical Parameters } \\
\hline$m_{1}$ & $4.168 \mathrm{~kg}$ & $c_{1}$ & $3.02 \mathrm{Ns} / \mathrm{m}$ \\
$k_{1}$ & $11.47 * 10^{3} \mathrm{~N} / \mathrm{m}$ & $m_{2}$ & $32 \mathrm{~g}$ \\
\hline \multicolumn{4}{c}{ Reduced Parameters } \\
\hline$\varepsilon$ & $0.76 \%$ & $\lambda$ & 1.80 \\
\hline
\end{tabular}

of two impacts per cycle should possess different Lyapunov exponents. This duration is shorter, positive Lyapunov exponents should be larger. For this reason, transient Lyapunov exponents of different response regimes with different $b$ are put in Table 3. With the increase of $b$, the duration of two impacts per cycle decreases and transient Lyapunov exponents increase, which verifies the initial assumption.

\section{Experimental identification of Lyapunov Exponents}

In $[9,10]$, the above-mentioned response regimes have been experimentally observed. Here, the objective is to explore the possibility of the identification of Lyapunov exponents from experimental data.

Firstly, a theoretical method to identify Lyapunov exponents from experimental data is presented. Then, it is applied to both a steady response with two impacts per cycle and a SMR with chaotic characteristics.

\subsection{Analytical development}

Based on the analytical development in Section 3, we propose a method here to identify Lyapunov exponents from experimental data in Ref. [9].

To calculate Lyapunov exponents of a specific response, matrix $J_{i}$ and $M_{i}$ should be estimated from experimental data. The common parameters in Table 4 can be used as the starting point of calculation, and other parameters should be identified from experimental data and they are further calculated to finish the estimation process. Specifically, to calculate $J_{i}$, only the impact time are further needed. Then to calculate $M_{i}$ in Eq. (27), the situation will be a little complex and some approximations will be used. Among the parameters needed to calculate $M_{i}, G$ is constant as follows:

$G=\left[\begin{array}{llll}0 & 1 & 0 & -1\end{array}\right]$

$S(\tau)$ will be a time variable if $R$ is not time invariant or has different values at different impact sides.
$S(\tau)=\left[\begin{array}{cccc}\frac{1-R \varepsilon}{\varepsilon+1} & 0 & \frac{\varepsilon+R \varepsilon}{\varepsilon+1} & 0 \\ 0 & 1 & 0 & 0 \\ \frac{1+R}{\varepsilon+1} & 0 & \frac{-R+\varepsilon}{\varepsilon+1} & 0 \\ 0 & 0 & 0 & 1\end{array}\right]$

$M_{i}$ is expressed in the following form:

$M_{i}=S\left(\tau_{i+1}\right)-\frac{\left[S\left(\tau_{i+1}\right) \dot{\Phi}\left(\tau_{i+1}^{-}\right)-\dot{\Phi}\left(\tau_{i+1}^{+}\right)\right] G}{G \dot{\Phi}\left(\tau_{i+1}^{-}\right)}$

Because $\ddot{y}(\tau)=0$, the state variables before and after impact are:

$\dot{\Phi}\left(\tau_{i+1}^{-}\right)=\left[\ddot{x}\left(\tau_{i+1}^{-}\right) \dot{x}\left(\tau_{i+1}^{-}\right) 0 \dot{y}\left(\tau_{i+1}^{-}\right)\right]$

$\dot{\Phi}\left(\tau_{i+1}^{+}\right)=\left[\ddot{x}\left(\tau_{i+1}^{+}\right) \dot{x}\left(\tau_{i+1}^{+}\right) 0 \dot{y}\left(\tau_{i+1}^{+}\right)\right]$

and

$G \dot{\Phi}\left(\tau_{i+1}^{-}\right)=\dot{x}\left(\tau_{i+1}^{-}\right)-\dot{y}\left(\tau_{i+1}^{-}\right)$

If $x, \dot{x}$ and $\ddot{x}$ are given, $y$ should meet the following requirement at any impact moments:

$\left|x\left(\tau_{i}\right)-y\left(\tau_{i}\right)\right|=b$

If the direction of relative displacement between LO and VI NES is changed, the following relation should hold:

$\left(x\left(\tau_{i}\right)-y\left(\tau_{i}\right)\right)\left(x\left(\tau_{i+1}\right)-y\left(\tau_{i+1}\right)\right)<0$

The reason why Eq. (40) is simplified to the form in Eq. (41) is that almost all relative displacement change direction in our experimental observations. If other cases are encountered, Eq. (40) can still be applied.

Supposing that the friction between VI NES and LO is small enough that it will not change the velocity of VI NES, then the following approximation relation between $y$ and $\dot{y}_{i}$ can be obtained:

$y\left(\tau_{i+1}\right)-y\left(\tau_{i}\right)=\dot{y}_{i}\left(\tau_{i+1}-\tau_{i}\right)$

where $\dot{y}_{i}$ is the velocity at $i^{\text {th }}$ time interval $\left[\tau_{i}, \tau_{i+1}\right]$, and combining Eqs. (40) and (42), $\dot{y}_{i}$ can be calculated:

$\dot{y}_{i}=\frac{x\left(\tau_{i+1}\right)-x\left(\tau_{i}\right) \pm 2 b}{\tau_{i+1}-\tau_{i}}$

With obtained $\dot{y}$, restitution coefficient $R$ can be calculated by Eq. (4). 


\subsection{Application}

To estimate Lyapunov exponents, an estimated constant restitution coefficient with $R=0.85$ is applied here, and this value of restitution coefficient is just an approximation to its real value and is just enough to demonstrate the proposed method here.

\subsubsection{Non-chaotic}

For $b=9 \mathrm{~mm}$, a steady response with two impacts per cycle is observed and its time history of acceleration is showed in Fig. 15, in which two impacts per cycle is well demonstrated.

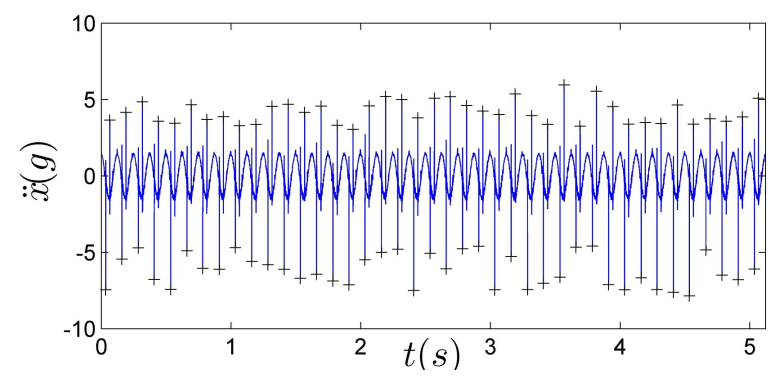

Fig. 15 Acceleration of LO with $b=0.009 \mathrm{~m}$ : impact moments are denoted by cross [8]

Its time history of displacement is showed in Fig. 16 and impacts moments are marked out by red circles. Then the velocities before and after impacts are estimated, and the corresponding phase portrait between displacement and velocity is showed in Fig. 17.

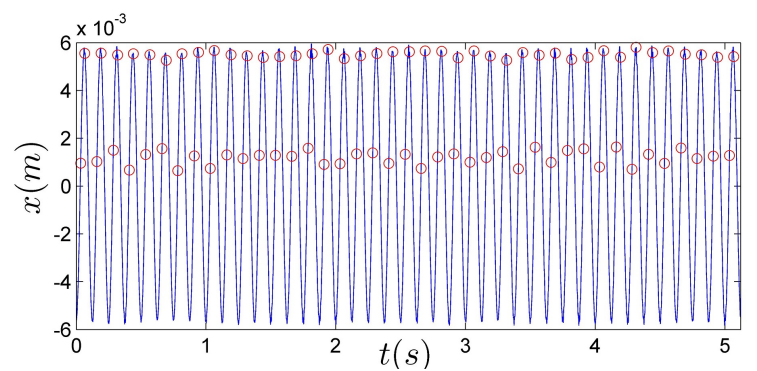

Fig. 16 Displacement of LO with $b=0.009 \mathrm{~m}$ : impact moments are denoted by circles

Because these values of displacements and velocities after and before impacts are so close that they cannot be clearly distinguished. To resolve this problem, the displacements and velocities after impact at the Poincare section with $\mid x-$ $y \mid=b$ is showed in Fig. 18 .

Then Lyapunov exponents are estimated during limited time with limited impact numbers, and the result is showed

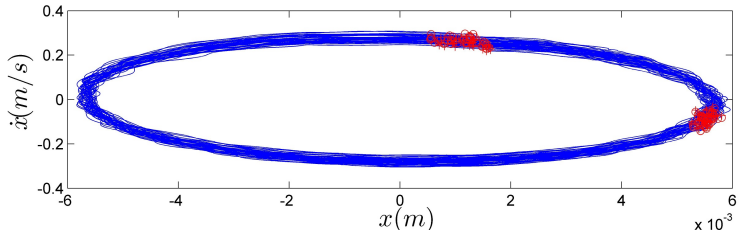

Fig. 17 Phase portrait of LO between displacement and velocity with $b=0.09 \mathrm{~m}$ : the moments before and after impact are denoted by red circles and crosses

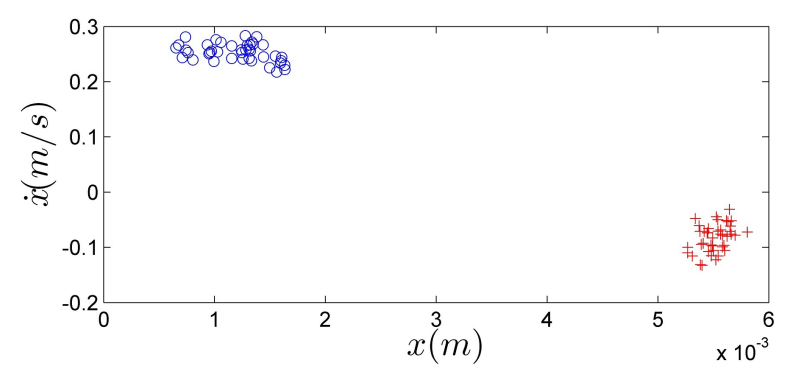

Fig. 18 Poincare section with $|x-y|=b$ : two groups of velocities and displacements of LO after impacts

in Fig. 19. Two facts are observed as follows: the transient Lyapunov exponents are positive in this short period and they are decreasing continuously. Compared to the results in Fig. 11 and 12, it can be anticipated that it will approach zero so long as the duration of time is long enough.

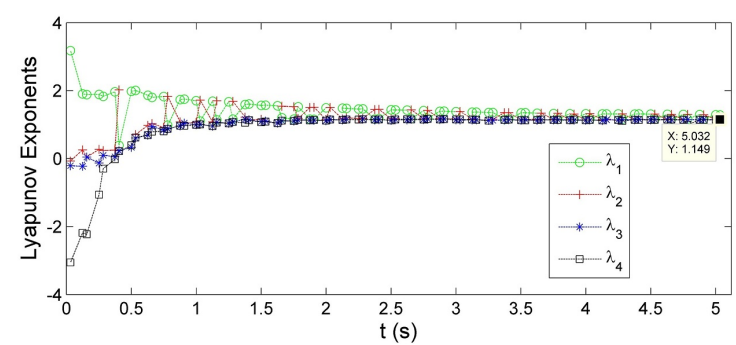

Fig. 19 Evolution of Lyapunov exponents of a steady response with two impacts per cycle for $b=0.009 \mathrm{~m}$

\subsubsection{Chaotic}

For $b=15 \mathrm{~mm}$, a chaotic response is observed. Its chaotic characteristic can be observed from different viewpoints. Its time history of acceleration is showed in Fig. 20, and a detailed analysis of its chaotic impact time difference can be found in [8].

Its time history of displacement is showed in Fig. 21 and impacts moments are marked out by red circles.

Then, the velocity is estimated from displacement and acceleration, and the corresponding phase portrait between displacement and velocity is showed in Fig. 22. The chaotic 


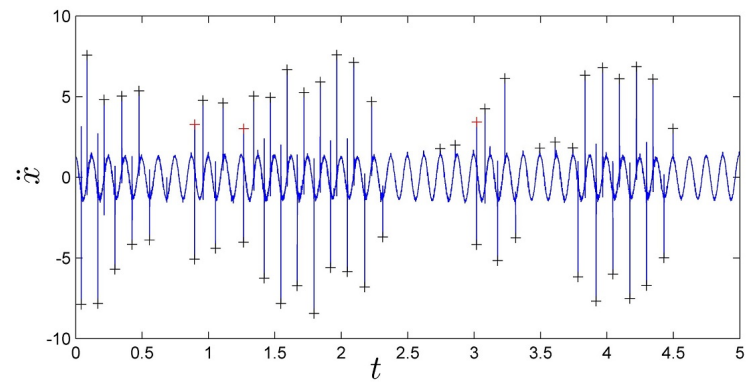

Fig. 20 Acceleration of LO with $b=0.015 \mathrm{~m}$ : impact moments are denoted by cross [8]

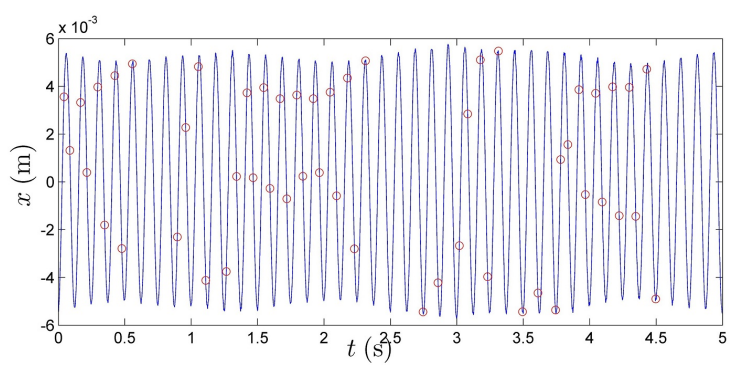

Fig. 21 Displacement of LO with $b=0.015 \mathrm{~m}$ : impact moments are denoted by circles

characteristic is well demonstrated by the irregular distribution of impacts.

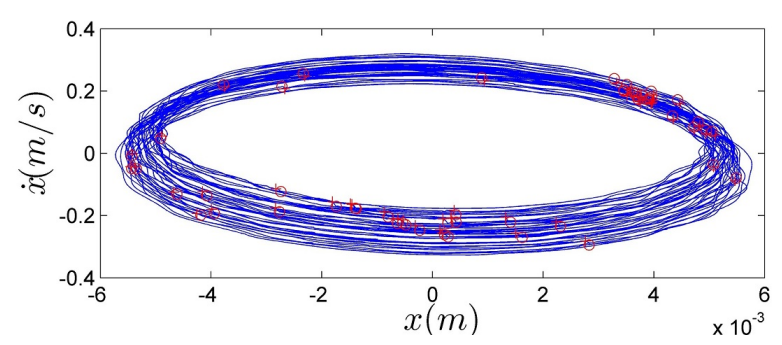

Fig. 22 Phase portrait of LO between displacement and velocity with $b=0.015 \mathrm{~m}$ : the moments before and after impact are denoted by red circles and crosses

Moreover, it is better demonstrated by the displacements and velocities after impacts at the Poincare section with $\mid x-$ $y \mid=b$ as showed in Fig. 23. Scattered points at both places are a well proof of chaos compared to these of the former periodic response in Fig. 18.

Then Lyapunov exponents are estimated during this limited time with limited impact numbers, and the result is showed in Fig. 24. Two facts are observed as follows: the transient Lyapunov exponents are positive and they are almost already stable. Compared to the results in Fig. 5, they are consistent.

It has to be pointed out that the same conclusion can be obtained for other restitution coefficients for the impacts at the two sides of LO or even different restitution coefficients at these two sides.

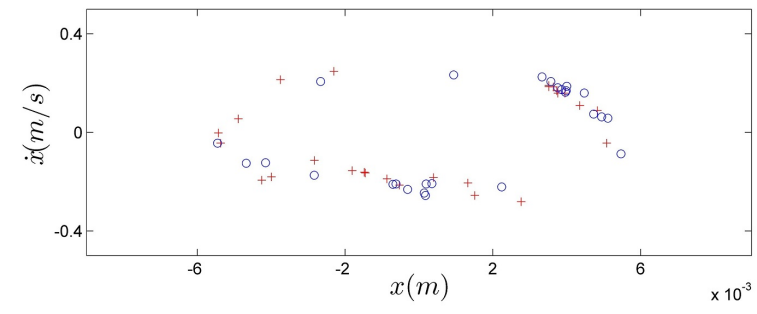

Fig. 23 Poincare section with $|x-b|=b$ : two groups of velocities and displacement of LO after impact

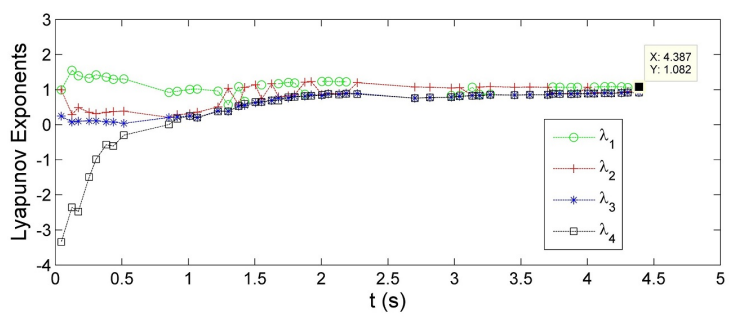

Fig. 24 Evolution of Lyapunov exponents of a SMR with $b=0.015 \mathrm{~m}$

\section{Conclusion}

In this paper, the method to calculate Lypapunov exponents of a vibro-impact system is theoretically derived. Combined with numerical simulation, the chaotic characteristics of different responses are studied. Finally, an approach to identify Lyapunov exponents from experimental data is proposed and is analyzed with some existing experimental data.

Lyapunov exponents for different response regimes is obtained and the chaotic characteristic is verified. In addition, it is found that the essence of chaotic is the irregular and desynchronized impacts, which is common base of the same chaotic feature of different response regimes. Therefore, although the overall effect reflected by Lyapunov exponents is important, the underlying factor that contributes to this average effect should be found. By this way, the relation between chaotic characteristic and other characteristic such as modulation and synchronization could be clearer. This point is more fundamental than Lyapunov exponents. This idea applies for other nonlinear systems with chaos.

Then, Lyapunov exponents are estimated for both a steady response with two impact per cycle and SMR, i.e. non-chaotic and chaotic response. Transient positive Lyapunov exponents within a limited time are obtained for both responses. A continuously decreasing positive value is obtained for nonchaotic response in this limited time and a steady positive value is obtained for SMR. Therefore, this response regime can be termed as chaotic SMR. The experimental results are consistent with what have been numerically obtained. But it has to be pointed out that the restitution coefficient will not be constant from an experimental viewpoint, and the used fixed value here is chosen as close to the fact as possible. The main error comes from different impact materials at two 
sides of LO and the neglected friction between VI NES and LO. The specific way to estimate restitution coefficient is out the scope of this paper and will be presented later.

Acknowledgements The authors acknowledge the French Ministry of Science and the Chinese Scholarship Council under grant No. 20130449 0063 for their financial support.

\section{References}

1. Ibrahim, R.A.: Vibro-impact dynamics: modeling, mapping and applications, vol. 43. Springer Science \& Business Media,, Berlin (2009)

2. Vakakis, A.F., Gendelman, O., Bergman, L., McFarland, D., Kerschen, G., Lee, Y.: Nonlinear targeted energy transfer in mechanical and structural systems, vol. 156. Springer Science \& Business Media, Berlin (2008)

3. Lee, Y., Nucera, F., Vakakis, A., McFarland, D., Bergman, L.: Periodic orbits, damped transitions and targeted energy transfers in oscillators with vibro-impact attachments. Physica D 238(18), 1868-1896 (2009)

4. Gendelman, O.V.: Analytic treatment of a system with a vibroimpact nonlinear energy sink. J. Sound Vib. 331, 4599-4608 (2012)

5. Gendelman, O., Alloni, A.: Dynamics of forced system with vibro-impact energy sink. J. Sound Vib. 358, 301-314 (2015)

6. Gendelman, O., Alloni, A.: Forced system with vibro-impact energy sink: Chaotic strongly modulated responses. Procedia IUTAM 19, 53-64 (2016)

7. Gourc, E., Michon, G., Seguy, S., Berlioz, A.: Targeted energy transfer under harmonic forcing with a vibro-impact nonlinear energy sink: Analytical and experimental developments. J. Vib. Acoust. 137(3), 031-008 (2015)

8. Li, T., Seguy, S., Berlioz, A.: Dynamics of cubic and vibro-impact nonlinear energy sink: Analytical, numerical, and experimental analysis. J. Vib. Acoust. 138(3), 031-010 (2016)

9. Li, T., Seguy, S., Berlioz, A.: On the dynamics around targeted energy transfer for vibro-impact nonlinear energy sink. Nonlinear Dyn. 87(3), 1453-1466 (2017)

10. Li, T., Seguy, S., Berlioz, A.: Optimization mechanism of targeted energy transfer with vibro-impact energy sink under periodic and transient excitation. Nonlinear Dyn. 87(4), 2415-2433 (2017)

11. Dynamics of two vibro-impact nonlinear energy sinks in parallel under periodic and transient excitations. Int. J. NonLinear Mech. 90, 100 - 110 (2017)

12. Pennisi, G., Stephan, C., Gourc, E., Michon, G.: Experimental investigation and analytical description of a vibro-impact nes coupled to a single-degree-of-freedom linear oscillator harmonically forced. Nonlinear Dyn. pp. 1-16 (2017). DOI 10.1007/s11071017-3344-1

13. Müller, P.C.: Calculation of lyapunov exponents for dynamic systems with discontinuities. Chaos Soliton. Fract. 5(9), 1671-1681 (1995)

14. Lamarque, C.H., Bastien, J.: Numerical study of a forced pendulum with friction. Nonlinear Dyn. 23(4), 335-352 (2000)

15. Stefanski, A.: Estimation of the largest lyapunov exponent in systems with impacts. Chaos Soliton. Fract. 11(15), 2443-2451 (2000)

16. Stefański, A., Kapitaniak, T.: Estimation of the dominant lyapunov exponent of non-smooth systems on the basis of maps synchronization. Chaos Soliton. Fract. 15(2), 233-244 (2003)

17. De Souza, S.L., Caldas, I.L.: Calculation of lyapunov exponents in systems with impacts. Chaos Soliton. Fract. 19(3), 569-579 (2004)
18. Jin, L., Lu, Q.S., Twizell, E.: A method for calculating the spectrum of lyapunov exponents by local maps in non-smooth impactvibrating systems. J. Sound Vib. 298(4), 1019-1033 (2006) 